\title{
Utility of Discography as a Preoperative Diagnostic Tool for Intradural Lumbar Disc Herniation
}

\author{
Tomiya Matsumoto ${ }^{1}$, Hiromitsu Toyoda ${ }^{2}$, Hidetomi Terai ${ }^{2}$, \\ Sho Dohzono ${ }^{2}$, Yusuke Hori ${ }^{2}$, Hiroaki Nakamura ${ }^{2}$ \\ ${ }^{1}$ Department of Orthopaedic Surgery, Osaka Rosai Hospital, Osaka, Japan \\ ${ }^{2}$ Department of Orthopaedic Surgery, Osaka City University Graduate School of Medicine, Osaka, Japan
}

Preoperative definitive diagnosis of intradural lumbar disc herniation (ILDH) is difficult despite the availability of various neuroradiological investigative tools. We present a case of ILDH diagnosed preoperatively by discography and computed tomography-discography (disco-CT).The patient was a 63-year-old man with acute excruciating right leg pain. Discography and disco-CT demonstrated leakage of the contrast medium into the intradural space. Based on these findings, a right L5 nerve root disturbance caused by ILDH was diagnosed. A right $\mathrm{L} 5$ hemi-laminectomy and a dorsal durotomy were performed. The herniated disc was carefully dissected and then completely removed. Three months after surgery, the patient had fully recovered. This report highlights the importance of making a definitive diagnosis of ILDH preoperatively for better surgical planning and improved clinical outcomes. Furthermore, discography and disco-CT are both useful preoperative diagnostic tools for the diagnosis of ILDH.

Keywords: Intradural lumbar disc herniation; Preoperative diagnosis; Discography

\section{Introduction}

Intradural lumbar disc herniation (ILDH) is rare, and the incidence is reported as $0.04 \%-0.33 \%$ of lumbar disc herniations (LDH) [1,2]. The first report of an intradural herniation was presented by Dandy [3] in 1942. Preoperative diagnosis of ILDH is still difficult despite the availability of newer neuroradiologic investigative tools, such as computed tomography (CT) and magnetic resonance imaging (MRI) [4]. In some reports, the diagnosis is confirmed intraoperatively [2]. The purpose of this report is to present a case of ILDH diagnosed preoperatively by discography, to discuss its differential diagnosis, and to review the relevant literature.

\section{Case Report}

\section{Presentation}

A 63-year-old man who experienced sudden onset of excruciating right leg pain, tingling, and numbness a week prior to admission was presented to our hospital. He had a history of LDH of L4-5 and had undergone conservative treatment at another hospital 5 years prior.

\section{Examination}

Neurological examination revealed sensory disturbances involving the L5 dermatome on the right side, and muscle weakness of the right tibialis anterior, extensor hallucis

\footnotetext{
Received Dec 8, 2015; Revised Jan 5, 2016; Accepted Jan 9, 2016

Corresponding author: Tomiya Matsumoto

Department of Orthopaedic Surgery, Osaka Rosai Hospital, 1179-3 Nagasonecho, Kita-ku, Sakai 591-8025, Japan

Tel: +81-72-252-3561, Fax: +81-72-255-3349, E-mail: tomiya@med.osaka-cu.ac.jp
} 
longus and peroneus longus muscle along with attenuated deep tendon reflexes. Straight leg raise test was positive at $20^{\circ}$ on the right side. Bladder and bowel functions were normal.

MRI revealed extrusion of disc material and abrupt loss of continuity of the dural sac at the L4-5 disc level as well as a small ovoid-shaped intradural lesion at the level of the right L5 pedicle (Fig. 1). Both T1- and T2-weighted MRI demonstrated an isointense lesion. A plain CT scan revealed the presence of gas in the L4-5 disc and in the spinal canal (Fig. 2). Discography and disco-CT demonstrated leakage of the contrast medium into the intradural space similar to potential findings on a myelographic study (Fig. 3). Based on these findings, the right L5 nerve root disturbance caused by ILDH was diagnosed and surgery was performed.

\section{Operation and postoperative course}

A right L5 hemi-laminectomy was performed with the patient in the prone position. No significant extradural lesion remained after the hemi-laminectomy. However, a hard mass could be palpated along the dural sac at the L4-5 disc level. The dural sac was swollen and immobile.
A dorsal 30-mm durotomy was performed in the midline. After the cauda equina nerve rootlets were carefully retracted, the herniated disc fragment was observed. Based
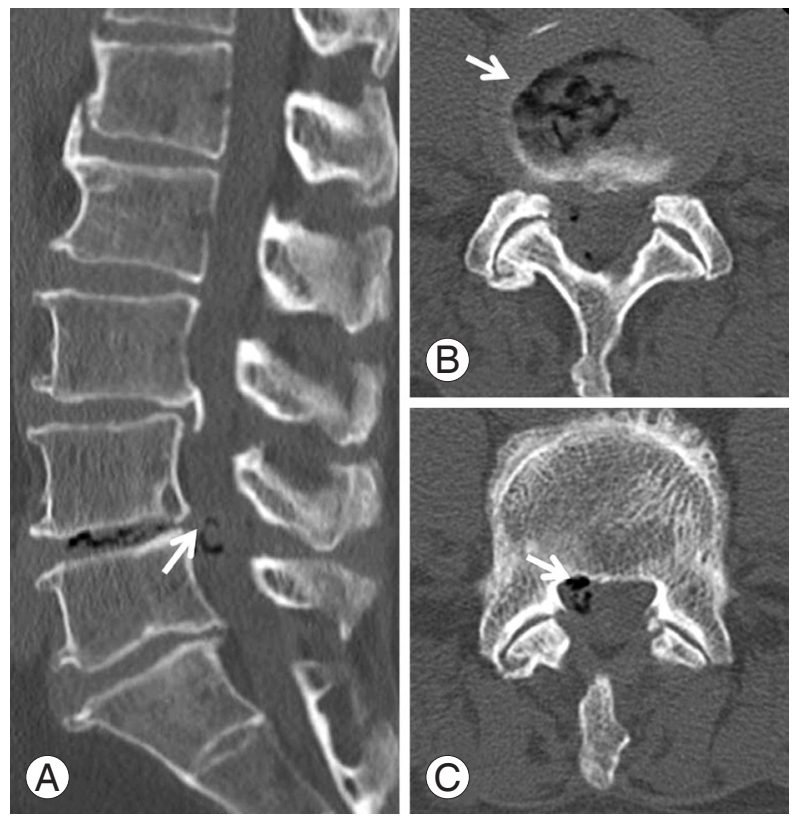

Fig. 2. Computed tomography (CT) scan. (A) Sagittal view. (B, C) Axial view. A plain CT scan revealed the presence of gas (arrow) at the $L 4-5$ disc and intraspinal canal.
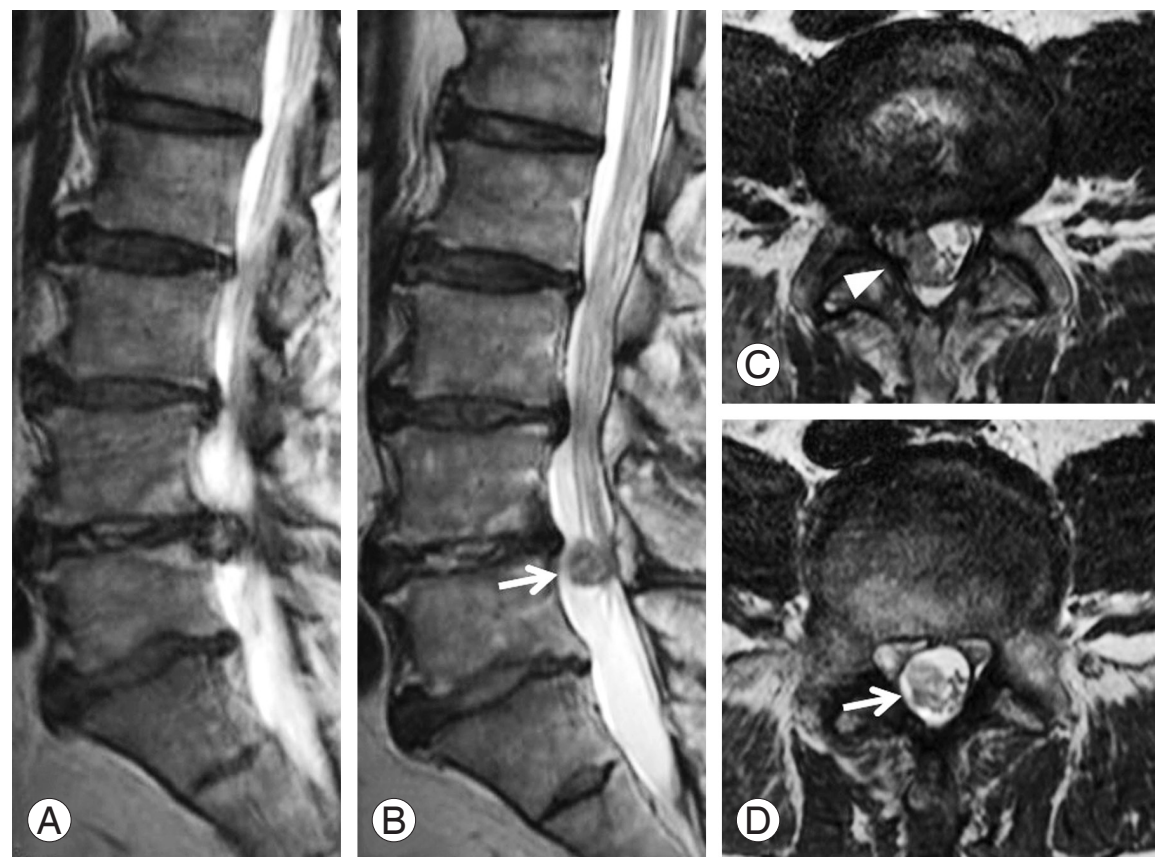

Fig. 1. T2-weighted magnetic resonance image (MRI). (A, B) Sagittal view. (C, D) Axial view. MRI revealed an extrusion of disc and abrupt loss of continuity of the dural sac (arrowhead) at the L4-5 disc level and a small ovoid-shaped intradural lesion (arrow) at the level of the right L5 pedicle. Both T1- and T2-weighted MRI demonstrated an isointense lesion. 
on these findings, we made a definitive diagnosis of ILDH. However, the surface of the intradural herniated disc fragment was totally smooth, and the sharp cartilaginous endplate was partially observed to be protruding from the herniated disc. The herniated disc fragment was carefully dissected and then completely removed under the surgical microscope (Fig. 4). The ventral dura and arachnoid membrane were adherent. After the intradural herniated disc fragment was removed, the ventral dural rent had ragged edges and was adherent to the L4-5 disc space. The ventral and dorsal dural rents were repaired with 6-0 nylon. Histological examination revealed that the lesion was disc material. The patient reported improvement in his radicular symptoms immediately after surgery, and the postoperative period was uneventful. Postoperative MRI showed complete removal of the intradural herniated disc (Fig. 5). The patient recovered fully after 3 months.
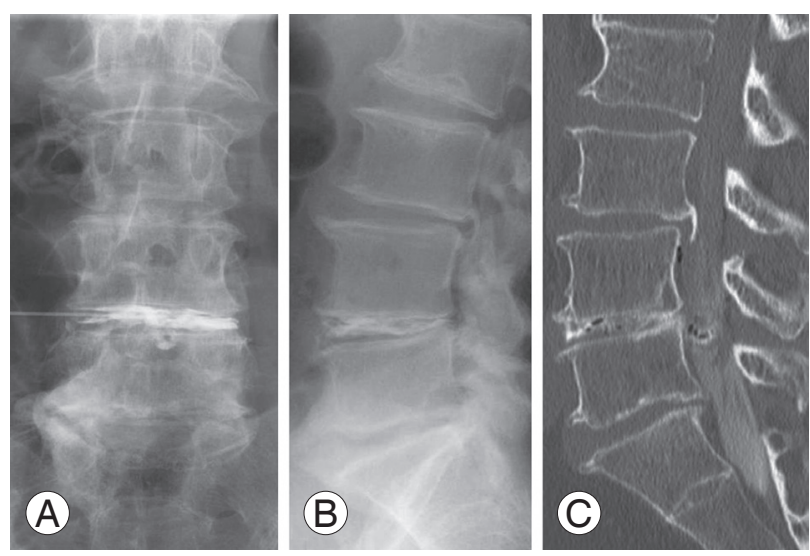

Fig. 3. Discography (A, B) and disco- computed tomography (CT) (C). (A) Posterior-anterior view. Contrast medium was injected into the L4-5 disc. (B, C) Sagittal view. Discography and disco-CT demonstrated leakage of the contrast medium into the intradural space.

\section{Discussion}

Intradural disc herniation is a rare condition and comprises $0.27 \%$ of all disc herniations [5,6], with about $3 \%$ occurring in the cervical region, $5 \%$ in the thoracic region, and $92 \%$ in the lumbar region [7]. The site most frequently affected by ILDH is L4-5 (51\%), followed by L3-4 (21\%), L5-S1 (11\%), and L2-3 and L1-2 [1].

Migration of the nucleus pulposus into the intradural site requires perforation of the annulus fibrosus, the posterior longitudinal ligament (PLL), and the dura mater [2]. The pathophysiology of ILDH is typically attributed to

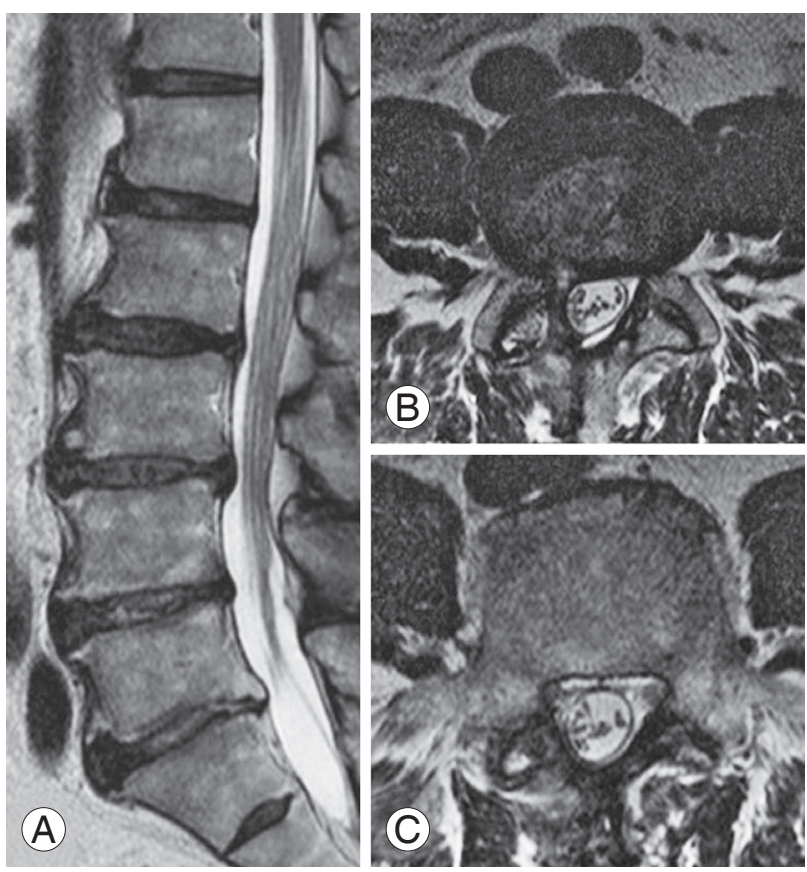

Fig. 5. T2-weighted magnetic resonance image (MRI). (A) Sagittal view. $(\mathbf{B}, \mathbf{C})$ Axial view. MRI revealed complete removal of the intradural hernia mass.
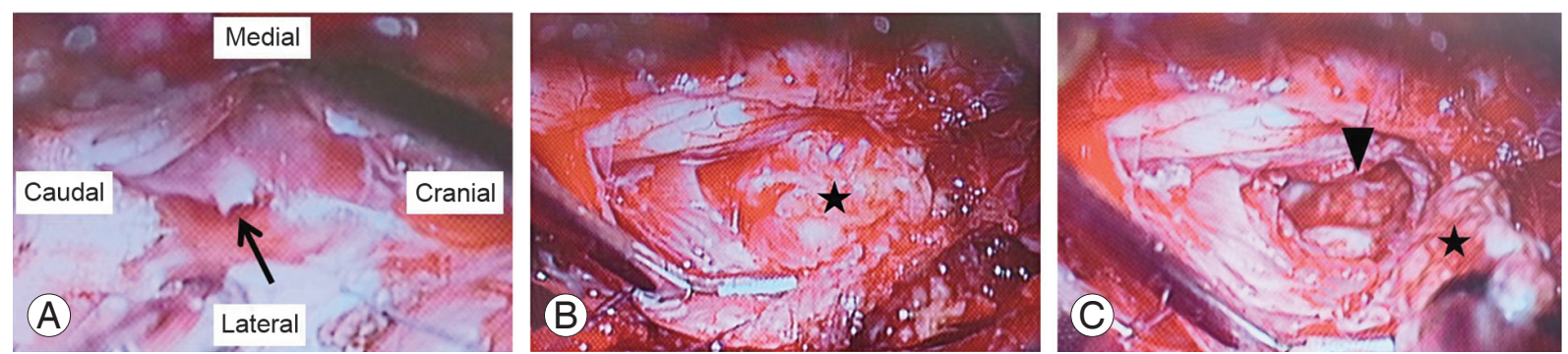

Fig. 4. Intraoperative imaging after opening the dural sac. (A) After the cauda equina nerve rootlets were retracted, the protrusion of the cartilaginous endplate from the herniated disc (arrow) was demonstrated. (B, C) After the herniated disc was incised, the herniated disc fragment (star) was demonstrated and a dural rent (arrowhead) was seen on the ventral dura after removal of the intradural herniated disc fragment. 
three general mechanisms: (1) congenital thickness of the dura mater and/or a union between the dura mater and the PLL; (2) local inflammation secondary to degenerative disc disease, which can contribute to the formation of adhesions between the dura mater and PLL, leading to spontaneous perforation into the intrathecal site; and (3) postoperative iatrogenic adhesions between the annulus fibrosus, the PLL, and the dura mater leading to perforation $[2,8,9]$. In the present case, the patient had a history of conservative treatment for LDH of L4-5 five years prior, and the operative findings revealed the protrusion of a sharp cartilaginous endplate from the intradural herniated disc. Therefore, adhesion of the dura mater, caused by chronic inflammation of the herniated disc, combined with a sharp cartilaginous endplate from the herniated disc may had led to occurrence of ILDH in this instance.

An important feature of ILDH is that it is difficult to definitively diagnose preoperatively. Although various imaging tools have been used in an attempt to preoperatively diagnose ILDH, affirmative diagnoses in previous studies have been made intraoperatively [2]. D'Andrea et al. [2] reviewed 122 cases of ILDH described in the previous literature, including their own cases, and reported that only 8 of 122 cases could have been diagnosed preoperatively. They stated that the radiological diagnosis of ILDH is possible in carefully selected patients but that a positive diagnosis can only be made through surgery.

Moreover, the prognosis of ILDH was not as easy to predict as the "typical" disc herniation. In about $67 \%$ of cases, satisfactory results are reported, while in $33 \%$ of cases, residual neurologic deficit, micturition urgency, sensory loss, and muscular atrophy remained [2].

We considered the difficulty with preoperative diagnosis as a reason for poor postoperative outcome. Choi et al. [10] reported that the postoperative complete neurologic deficit (foot drop) was the result of nerve damage caused by several intraoperative attempts to manipulate the dural sac, possibly because the intradural disc herniation went unnoticed. Therefore, preoperative diagnosis is thought to be absolutely critical for the prevention of postoperative neurologic deficits and for the improvement of postoperative clinical outcomes.

Various neuroradiological investigative tools could be used for diagnosing ILDH. Myelography has not been useful because the myelographic finding of intradural disc herniation is not specific. In about $65 \%$ of cases, the finding is of a total myelographic block [11], and it is not al- ways possible to determine an intra- or extradural origin. Hidalgo-Ovejero et al. [8] pointed out the significance of gas within the spinal canal associated with intradural disc herniations. They concluded that the potential presence of ILDH must always be considered in a patient whose CT study shows the presence of epidural gas. In the present case, a plain CT revealed the presence of gas inside the L4-5 disc and within the spinal canal.

MRI findings of ILDH are important for its preoperative identification. Choi et al. [10] suggested that MRI findings demonstrating loss of continuity of the PLL and a sharp, beak-like appearance on T2-weighted images should be considered as indicators for potential presence of an ILDH preoperatively. Floeth reported that the differentiation of an intraspinal/intradural sequester versus an intraspinal/intradural tumorous or cystic space-occupying lesion is usually possible with current high-resolution MRI techniques [12]. The rim enhancement of the herniated disc on a gadolinium-enhanced MRI has been accepted as a typical MRI finding of ILDH [13]. However, the ring enhancement is a result of granulated tissue infiltrating the disc fragment over a long period and acute herniated discs show no rim enhancement [14]. In the present case, it was unclear if a loss of continuity of the PLL and a beaklike appearance on T2-weighted image were present, but MRI showed an abrupt loss of continuity of the dural sac and an intradural tumor-like lesion on the axial view on the T2-weighted image.

There have been very few reports about discography for preoperative ILDH diagnosis. Benyamin et al. [9] reported a case of ILDH incidentally diagnosed during routine discography. It was noted that contrast was not contained within the disc and spread intrathecally with a myelographic appearance during discography. In the present case, we suspected ILDH based on CT and MRI findings, and we performed discography and disco-CT to make a definitive diagnosis. Also, by performing discography and disco-CT, we could distinguish ILDH from other spinal pathologies that must be considered in the differential diagnosis, such as neurofibroma, lipoma, meningioma, epidermoid tumor, arachnoid cyst, arachnoiditis, and metastasis. However, differentiation of these other pathologic causes of pain from ILDH has previously been reported to be difficult $[4,15]$.

This report highlights the fact that it is very important to make a definitive diagnosis of ILDH preoperatively, both for better surgical planning and for improved clini- 
cal outcomes. Additionally, discography and disco-CT are useful preoperative diagnostic tools for ILDH. Therefore, we strongly suggest that discography and disco-CT should be performed when ILDH is being considered based upon initial MRI and CT findings.

\section{Conflict of Interest}

No potential conflict of interest relevant to this article was reported.

\section{References}

1. Kataoka O, Nishibayashi Y, Sho T. Intradural lumbar disc herniation: report of three cases with a review of the literature. Spine (Phila Pa 1976) 1989;14:529-33.

2. D’Andrea G, Trillo G, Roperto R, Celli P, Orlando ER, Ferrante L. Intradural lumbar disc herniations: the role of MRI in preoperative diagnosis and review of the literature. Neurosurg Rev 2004;27:75-80.

3. Dandy WE. Recent advances in the diagnosis and treatment of ruptured intervertebral disks. Ann Surg 1942;115:514-20.

4. Liu CC, Huang CT, Lin CM, Liu KN. Intradural disc herniation at L5 level mimicking an intradural spinal tumor. Eur Spine J 2011;20 Suppl 2:S326-9.

5. Koc RK, Akdemir H, Oktem IS, Menku A. Intradural lumbar disc herniation: report of two cases. Neurosurg Rev 2001;24:44-7.

6. Oztürk A, Avci E, Yazgan P, Torun F, Yucetas S, Karabag H. Intradural herniation of intervertebral disc at the level of Lumbar 1-Lumbar 2. Turk Neurosurg 2007;17:134-7.

7. Epstein NE, Syrquin MS, Epstein JA, Decker RE.
Intradural disc herniations in the cervical, thoracic, and lumbar spine: report of three cases and review of the literature. J Spinal Disord 1990;3:396-403.

8. Hidalgo-Ovejero AM, Garcia-Mata S, Gozzi-Vallejo S, Izco-Cabezon T, Martinez-Morentin J, MartinezGrande M. Intradural disc herniation and epidural gas: something more than a casual association? Spine (Phila Pa 1976) 2004;29:E463-7.

9. Benyamin R, Vallejo R, Yousuf N, Tamrazi A, Kramer $\mathrm{J}$. Incidental diagnosis of intradural lumbar disc herniation during discography: a case report. Pain Pract 2007;7:332-6.

10. Choi JY, Lee WS, Sung KH. Intradural lumbar disc herniation: is it predictable preoperatively? A report of two cases. Spine J 2007;7:111-7.

11. Paini GP, Baldi PG, Barra N, Pasetti S. Intradural rupture of an intervertebral disk. Ateneo Parmense Acta Biomed 1978;49:189-96.

12. Floeth F, Herdmann J. Chronic dura erosion and intradural lumbar disc herniation: CT and MR imaging and intraoperative photographs of a transdural sequestrectomy. Eur Spine J 2012;21 Suppl 4:S453-7.

13. Whittaker CK, Bernhardt M. Magnetic resonance imaging shows gadolinium enhancement of intradural herniated disc. Spine (Phila Pa 1976) 1994;19:1505-7.

14. Mailleux R, Redant C, Milbouw G. MR diagnosis of transdural disc herniation causing cauda equine syndrome. JBR-BTR 2006;89:303-5.

15. Konishi S, Nakamura H, Kato M, Toyoda H, Dozono M. Cauda equina tumor mimicking an intradural disc herniation, with emphasis on differential diagnosis: a case report. Acta Neurol Belg 2008;108:1679. 